\title{
Study of Few-Body Problems at WASA — the ABC Phenomenon
}

\author{
M. Bashkanov for the WASA-at-COSY Collaboration, ${ }^{\text {a }}$ \\ Physikalisches Institut der Universität Tübingen, Auf der Morgenstelle 14, D-72076 Tübingen
}

\begin{abstract}
A short overview over the ongoing few-body program at WASA@COSY is given with special emphasis on the $\mathrm{ABC}$ phenomenon, which is known since 50 years without having found a consistent explanation. We show that this phenomenon is linked with a resonant energy dependence in the total cross section of isoscalar double-pionic fusion processes. This resonance structure is connected with the baryon-baryon system, in particular with the $p n$ and $\Delta \Delta$ systems, however, possesses a mass, which is $90 \mathrm{MeV}$ below the nominal $\Delta \Delta$ threshold and has a width of only $50 \mathrm{MeV}, i$. e., five times smaller than expected from a conventional $t$-channel $\Delta \Delta$ process.
\end{abstract}

\section{Introduction}

The experimental program of WASA-at-COSY focuses on production and decay of meson and baryon systems, which arise from initial nucleon-nucleon collisions. This provides the possibility to span the research issues from fundamental questions about symmetry breaking and exotic processes in rare meson decays to the formation of exotic states in the production of few-nucleon systems. These include decays of $\eta, \omega$ and $\eta^{\prime}$, where experimental results reveal substantial discrepancies to NNLO ChPT predictions [1], as well as isospin breaking in the $d d \rightarrow \alpha \pi^{0}$ reaction, $\eta$-mesic nuclei and the ABC-effect. The latter has been a puzzle since 50 years. It deals with the observation of a low-mass enhancement in the $\pi \pi$ invariant mass in double-pionic fusion reactions leading to few-nucleon systems. This contribution concentrates on the ABC-effect in the most basic system, the $p n \rightarrow d \pi^{0} \pi^{0}$ reaction, where new exclusive high-statistics measurements have been carried out with WASA@COSY.

\section{Experiments and their Results}

Single and multi-pion production as well as $\eta, \omega, \eta^{\prime}$ and $K$ production in nucleon-nucleon collisions have been studied systematically in recent years at the proton storage rings CELSIUS and COSY. In two-pion production initiated by nucleon-nucleon collisions the single-nucleon excitation is favored at energies near threshold. Since single- $\Delta$ excitation is highly suppressed, single-Roper excitation dominates the near-threshold $\pi \pi$ production. In fact, the respective measurements conducted at CELSIUS/WASA [24] with the WASA detector setup [5] and at COSY-TOF [6] give results for the Roper resonance properties, which agree well with most recent results from analyses of photoand pion-induced reactions[7].

\footnotetext{
a e-mail: bashkano@pit.physik.uni-tuebingen.de
}

At higher energies double-nucleon excitations come into play. For $T_{p}>1 \mathrm{GeV}$ the $\Delta \Delta$ excitation gets the leading process [8] as can be observed in the respective $N \pi$ invariant mass spectra of $N N \pi \pi$ exit channels. Whereas this is at least in good qualitative - and partly in quantitative - agreement with theoretical predictions, we find a profound disagreement with such predictions for the case, where the isoscalar two-pion production leads to a nuclear bound system in the final state. This observation brings us back to the long-standing puzzle of the so-called ABCeffect. The acronym stands for an unexpected enhancement at low masses in the invariant $\pi \pi$ mass spectrum $M_{\pi \pi}$ first observed by Abashian, Booth and Crowe [9] in the double pionic fusion of deuterons and protons to ${ }^{3} \mathrm{He}$. Follow-up experiments [10-20] revealed this effect to be of isoscalar nature (with regard to the $\pi \pi$ system) and to show up only in cases, when the two-pion production process leads to a bound nuclear system. With the exception of low-statistics bubble-chamber measurements all previous experiments conducted on this issue have been inclusive measurements carried out preferentially with single-arm magnetic spectrographs for the detection of the fused nuclei.

Initially the low-mass enhancement had been interpreted as an unusually large $\pi \pi$ scattering length and evidence for the $\sigma$ meson, respectively [9]. Since the effect showed up particularly clear at beam energies corresponding to the excitation of two $\Delta \mathrm{s}$ in the nuclear system, the ABC effect was interpreted later on by a $t$-channel $\Delta \Delta$ excitation in the course of the reaction process leading to both a lowmass and a high-mass enhancement in isoscalar $M_{\pi \pi}$ spectra [21-26].

The first exclusive double-pionic fusion measurements of solid statistics carried out at CELSIUS/WASA revealed a number of remarkable features:

- First of all, in none of the investigated reactions $p n \rightarrow$ $d \pi^{0} \pi^{0}$ [28], pd $\rightarrow{ }^{3} \mathrm{He} \pi^{0} \pi^{0}$ [29], pd $\rightarrow{ }^{3} \mathrm{He} \pi^{+} \pi^{-}$[29], $d d \rightarrow{ }^{4} \mathrm{He} \pi^{0} \pi^{0}$ [30] and $d d \rightarrow{ }^{4} \mathrm{He} \pi^{+} \pi^{-}$[30] a significant high-mass enhancement is observed in the $\pi \pi$ invariant mass spectrum - contrary to what was recorded 

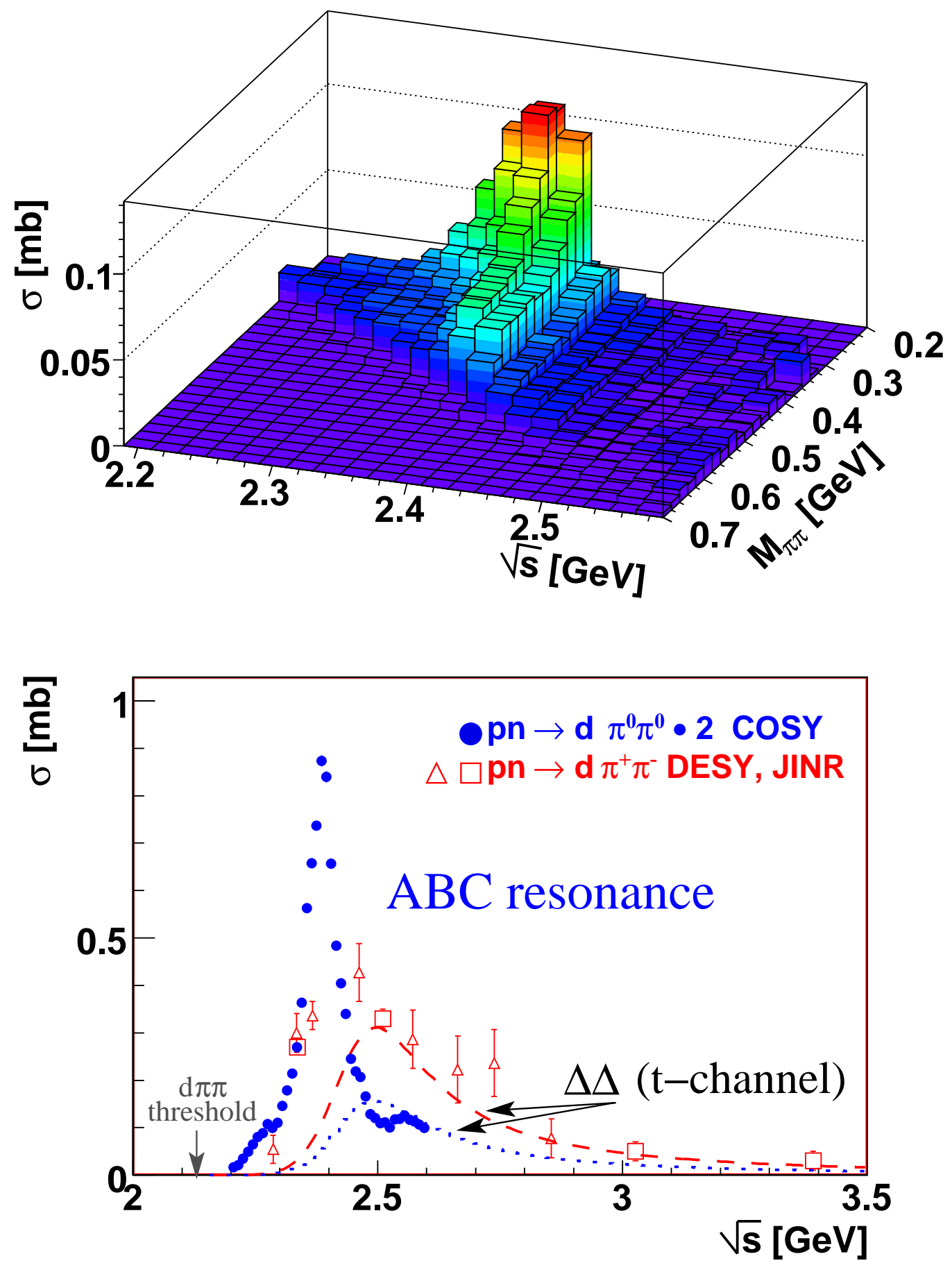

Fig. 1. Top: Energy dependence of $\pi^{0} \pi^{0}$ invariant mass $M_{\pi^{0} \pi^{0}}$ shown by a 3D-plot of $M_{\pi^{0} \pi^{0}}$ versus the total energy in the center-of-mass system $\sqrt{s}$. Bottom: : Energy dependence of the total cross section for the $p n \rightarrow d \pi^{+} \pi^{-}$reaction from threshold up to $\sqrt{s}=3.5 \mathrm{GeV}$. Data for the $d \pi^{+} \pi^{-}$channel are from JINR Dubna (squares) and DESY (open triangles). The results of this work for the $\pi^{0} \pi^{0}$ channel are scaled by the isospin factor of two and are given by the full circles. Dashed and dotted lines represent $t$-channel $\Delta \Delta$ calculations for $\pi^{+} \pi^{-}$ and $\pi^{0} \pi^{0}$ channels, respectively. From [33]

in inclusive measurements with single-arm magnetic spectrometers and also contrary to the theoretical predictions.
- Second, the most basic double-pionic fusion process, the $p n \rightarrow d \pi^{0} \pi^{0}$ reaction, exhibits a peculiar resonancelike behavior $[27,28]$ in the energy dependence of the 
$19^{\text {th }}$ International IUPAP Conference on Few-Body Problems in Physics

total cross section, which indicates a resonance in $p n$ and $\Delta \Delta$ systems at a mass roughly $100 \mathrm{MeV}$ below the mass of two $\Delta$ states and a width much smaller than expected from a conventional $t$-channel $\Delta \Delta$ excitation process.

- Third, the isovector double-pionic fusion process $p p \rightarrow$ $d \pi^{+} \pi^{0}$ does not exhibit any ABC effect $[31,32]-$ as also expected due to the fact that Bose symmetry excludes relative s-waves in the isosvector $\pi \pi$ system. Hence this channel is well described by the conventional $t$-channel $\Delta \Delta$ process.

In order to shed more light onto this issue we have measured the double-pionic fusion processes to $\mathrm{D},{ }^{3} \mathrm{He}$ and ${ }^{4} \mathrm{He}$ exclusively over practically the full phase space at WASA@COSY with orders of magnitude higher statistics than in the previous measurements. For the most basic fusion process, the one leading to deuterium, first results [33] from the data analysis are now available, see Fig. 1: At the top the spectrum of the $\pi^{0} \pi^{0}$-invariant mass $M_{\pi^{0} \pi^{0}}$ is shown in dependence of total energy in the center-of-mass system. Our data exhibit a striking low-mass enhancement, however, no apparent high-mass enhancement as predicted by conventional $\Delta \Delta$ calculations. Moreover, in the total cross section (Fig. 1, bottom) we observe an narrow resonancelike structure - again in contradiction to the conventional $\Delta \Delta$ process (dotted curve for the $\pi^{0} \pi^{0}$ channel - and dashed curve for the $\pi^{+} \pi^{-}$channel). As we see from Fig. 1, the ABC-effect, i.e. the low-mass enhancement in the $\pi \pi$-mass spectrum is present only at energies $\sqrt{s}$ within this narrow resonance structure, which has its maximum $90 \mathrm{MeV}$ below the $\Delta \Delta$ mass and a width of only $50 \mathrm{MeV}$, i.e. five times smaller than expected from the conventional $t$-channel $\Delta \Delta$ excitation. Indeed, describing this structure by a $s$-channel ansatz leads to a surprisingly good description of both the total and the differential distributions including the $\mathrm{ABC}$ effect in the $M_{\pi^{0} \pi^{0}}$ spectra.

Such a baryon-baryon resonance has been predicted by various quark-model calculations [34-39], some of which even predict this resonance to be a member of a dibaryon multiplet.

From the fact that the $\mathrm{ABC}$ effect is observed also for double-pionic fusion processes to heavier nuclei [13-18, $29,30,40]$, we conclude that this resonance is obviously robust enough to survive even in the nuclear medium.

\section{Acknowledgments}

We acknowledge valuable discussions with L. Alvarez-Ruso, Ch. Hanhart, E. Oset, F. Wang and C. Wilkin. This work has been supported by BMBF (06TU261, 06TU9193), the Forschungszentrum Jülich (COSY-FFE) and DFG (Europ. Graduiertenkolleg 683).

\section{References}

1. C. Adolph et al., Phys. Lett. B677, (2009) 24; arXiv: 0811.2763 [nucl-ex]
2. W. Brodowski et al., Phys. Rev. Lett. 88, (2002) 192301

3. J. Pätzold et al., Phys. Rev. C67, (2003) 052202(R)

4. T. Skorodko et al., Eur. Phys. J. A35, (2008) 317

5. Chr. Bargholtz et al., Nucl. Instr. Meth A594, (2008) 339

6. S. Abd El-Bary et al., Eur. Phys. J. A37, (2008) 267

7. A. V. Sarantsev et al., Phys. Lett.B659, (2008) 94; arXiv: 0707.3591 [hep-ph]

8. T. Skorodko et al., Phys. Lett.B679 (2009) 30 ; arXiv: 0906.3087 [nucl-ex] and references therein

9. A. Abashian, N. E. Booth, and K. M. Crowe, Phys. Rev. Lett. 5, (1960) 258

10. R. J. Homer et al., Phys. Rev. Lett.9, (1964) 72

11. J. H.Hall et al., Nucl. Phys. B12, (1969) 573

12. I. Bar-Nir et al., Nucl. Phys. B54,(1973) 17

13. J. Banaigs et al., Nucl. Phys. B67, (1973) 1

14. J. Banaigs et al., Nucl. Phys. B105, (1976) 52

15. F. Plouin et al., Nucl. Phys. A302, (1978) 413

16. A. Abdivaliev et al., Sov. J. Nucl. Phys.29, (1979) 796

17. F. Plouin, P. Fleury, C. Wilkin, Phys. Rev. Lett. 65, (1990) 690

18. R. Wurzinger et al., Phys. Lett. B445, (1999) 423

19. for a review see A. Codino and F. Plouin, $\mathrm{LNS} / \mathrm{Ph} / 94-$ 06

20. S. Dymov et al. Phys. Rev. Lett. 102, (2009) 192301 (1963)

21. T. Risser and M. D. Shuster, Phys. Lett. 43B, (1973) 68

22. I. Bar-Nir, T. Risser, M. D. Shuster, Nucl. Phys. B87, (1975) 109

23. J. C. Anjos, D. Levy, A. Santoro, Nucl. Phys. B67, (1973) 37

24. see, e.g., A. Gardestig, G. Fäldt, C. Wilkin, Phys. Rev. C59, (1999) 2608 and Phys. Lett. B421, (1998) 41

25. C. A. Mosbacher, F. Osterfeld, nucl-th/9903064

26. L. Alvarez-Ruso, Phys. Lett. B452, (1999) 207; PhD thesis, Univ. Valencia 1999

27. H. Clement et al., Prog. Part. Nucl. Phys. 61, (2008) 276

28. M. Bashkanov et al., Phys. Rev. Lett. 102, (2009) 052301; arXiv: 0806.4942 [nucl-ex]

29. M. Bashkanov et al., Phys. Lett. B637, (2006) 223

30. S. Keleta et al., Nucl. Phys. A825, (2009) 81

31. F. Kren et al., Proc. MESON08, Int. J. Mod. Phys. A24, (2009) 561

32. F. Kren et al., sumitted for publication; arXiv:0910.0995 [nucl-ex]

33. M. Bashkanov et al., Proc. PANIC08 (Elsevier, eds. I. Tserruya, A. Gal, D. Ashery), (2009) 239; arXiv: 0906.2328 [nucl-ex]

34. J. Ping et al., Phys. Rev. C65, (2002) 044003 and references therein; arXiv:0806.0458

35. T. Barnes et al., Phys. Rev. C48, (1993) 539

36. T. Kamae and T. Fujita, Phys. Rev. Lett. 38, (1977) 471

37. M. Oka, K. Yazaki, Prog. Theor. Phys. 66, (1981) 572

38. V. I. Kukulin et al., Nucl. Phys. A689, (2001) 327c

39. R. D. Mota et al., Phys. Rev. C65, (2002) 034006

40. A. Pricking et al., contribution to this conference 